\title{
Motivation in vigilance: A test of the goal-setting hypothesis of the effectiveness of knowledge of results*
}

\author{
JOEL S. WARM, SHERYL W. RIECHMANN, \\ ANTHONY F. GRASHA and BARBARA SEIBEL \\ University of Cincinnati, Cincinnati, Ohio 45221
}

This study tested the prediction, derived from the goal-setting hypothesis, that the facilitating effects of knowledge of results (KR) in a simple vigilance task should be related directly to the level of the performance standard used to regulate KR. Two groups of Ss received dichotomous $K R$ in terms of whether $S$ 's response times (RTs) to signal detections exceeded a high or low standard of performance. The aperiodic offset of a visual signal was the critical event for detection. The vigil was divided into a training phase followed by testing, during which $\mathrm{KR}$ was withdrawn. Knowledge of results enhanced performance in both phases. However, the two standards used to regulate feedback contributed little to these effects.

Knowledge of results (KR) has been found consistently to enhance vigilance performance. When the discriminations to be made are simple, these effects have been interpreted as primarily motivational rather than instructional in nature (Mackworth, 1970). Support for this position comes from studies indicating that noncontingent $\mathrm{KR}$ also facilitates vigilance performance (Loeb \& Schmidt, 1963) and that the partial reinforcement effect is observable in a vigilance task when KR is varied as the incentive (Warm, Hagner, \& Meyer, 1971).

Locke and his co-workers have considered the incentive value of KR in a variety of tasks in terms of a goal-setting hypothesis (Locke, 1968; Locke, Cartlege, \& Koeppel, 1968). They maintain that KR provides $S$ with specific standards by which to assess his performance and that the effects of KR are mediated by the goals that $\mathrm{S}$ sets in response to such knowledge. In the absence of KR, $S$ has no specific standard that could be used for goal setting and, therefore, less incentive to maintain a high level of effort.

According to Locke (1968), one implication from the goal-setting hypothesis is that the facilitative effects of KR should be related directly to the level of the performance standard used to regulate feedback. Within reasonable limits, the higher the standard, the greater the difficulty of the goal that $S$ will strive to achieve, and the greater the efficiency of his performance. The present study was designed, in part, to test this prediction within the context of a simple vigilance task.

In addition to enhancing overall vigilance

*This research was supported by the Institute of Space Sciences of the University of Cincinnati under National Aeronautics and Space Administration Grant No. NGL-36-004-014. The authors are indebted to Dr. F. H. Kanfer for his helpful comments. performance, KR has been shown to have residual properties that persist from training to subsequent testing in the absence of KR (Davies \& Tune, 1970). A second aspect of this investigation was to determine if characteristic differences could be identified in the residual properties of $\mathrm{KR}$ when feedback was given in relation to different standard levels of performance.

\section{METHOD}

Seventy-five college students, 64 men and 11 women, served as Ss. None had participated previously in a vigilance study. They monitored a display consisting of a single red jewel light, centered within a $13 \times 18 \mathrm{~cm}$ flat-black panel. Normally the light was on; its offset was the critical signal for detection. Activation by $S$ of a pushbutton switch, located immediately below the display lamp, reilluminated the lamp at offset. The display was mounted slightly below eye level, approximately $71 \mathrm{~cm}$ from the seated S.

Twenty-five Ss were assigned at random to one of three groups (with the restriction that 21 or 22 men appear in each group). Dichotomous KR was given to the $S s$ in two of the groups in terms of whether each response was faster or slower (and, therefore, better or worse) than an indicated standard of performance. In one of these groups, best-KR, the indicated performance standard was S's best previous performance, i.e., his fastest prior response. In the other group, worst-KR, the indicated standard was S's worst previous performance, i.e., his slowest prior response. The remaining group, acknowledgment, served as a control. Ss in this group received no evaluative feedback during the session except acknowledgment of each response by $\mathrm{E}$.

Knowledge of results and acknowledgment were provided by a console adjacent to the vigilance display. The console consisted of three lamps (red, amber, and green) arrayed along a horizontal vector. They were labeled "slower," "acknowledgment," and "faster," respectively. The red and green lamps were exposed for the two KR groups but only the amber lamp for the control group.

All Ss participated in an $85-\mathrm{min}$ session, divided into a $30-\mathrm{min}$ training phase and a 45-min testing phase. Acknowledgment and $\mathrm{KR}$ were provided only during training. A $10-\mathrm{min}$ rest period separated both phases. Each phase was divided into 15-min periods, during which critical signals were presented at an average rate of one/minute. Intersignal intervals ranged from 10 to $110 \mathrm{sec}$. Once presented, the signals remained on until terminated by S's response. Signal sequences were programmed at random and presented automatically with appropriate switching circuitry. Response times (RTs) to signal detections were recorded automatically to the nearest $.001 \mathrm{sec}$. Each $\mathrm{S}$ was run individually in a sound-shielded room; the control apparatus was located in an adjacent room. Further details concerning the apparatus and the task are presented in Warm et al (1972).

\section{RESULTS}

Median RTs were computed from the data of each S for each period of watch during training and testing. Means of median RTs for the three groups of Ss are plotted as a function of periods in Fig. 1. Data for the training and testing phases are presented separately in each panel. 


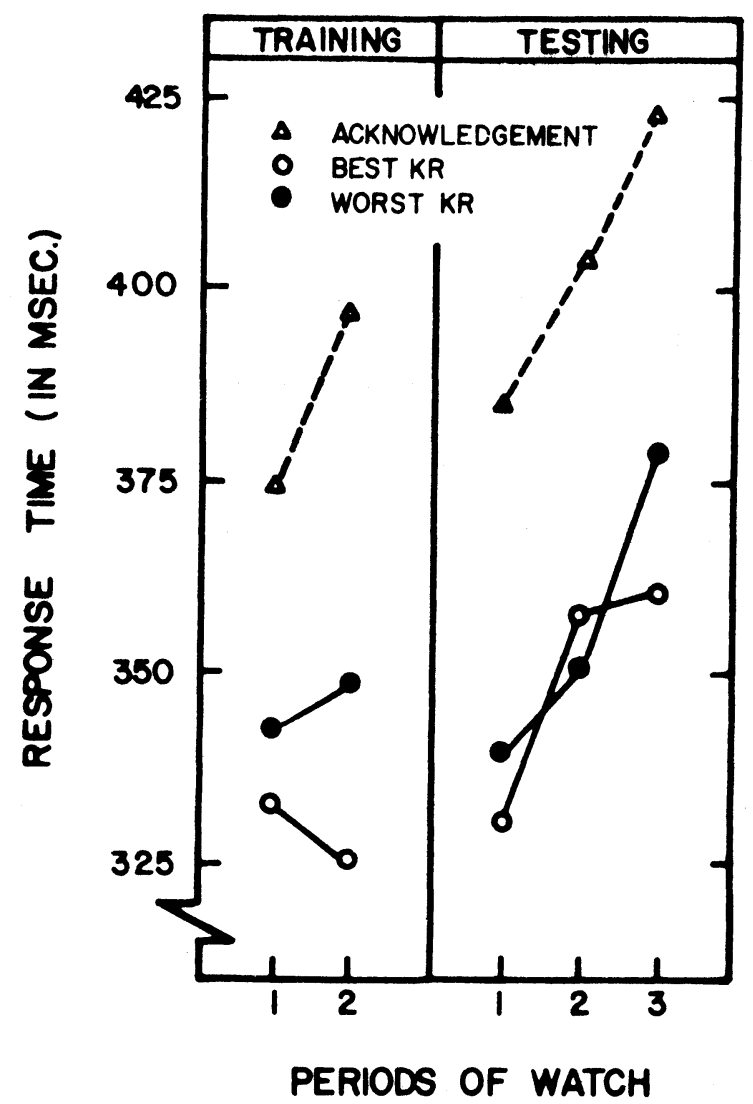

Fig. 1. Response time as a function of periods of watch $(15 \mathrm{~min})$ for all groups. Data for the training and transfer phases are plotted separately in each panel.

An analysis of variance of the training data revealed significant differences between groups $(\mathrm{F}=6.42$, $\mathrm{df}=2 / 72, \mathrm{p}<.005)$. Subsequent Newman-Keuls tests $(\alpha=.05)$ specified the locus of this effect; the RTs of both KR groups were faster than those of the acknowledgment group but did not differ significantly from each other. None of the remaining sources of variance were significant $(\mathrm{p}>.10)$. A similar analysis of the testing data also showed significant differences in RT between groups $(\mathrm{F}=4.88, \mathrm{df}=2 / 72, \mathrm{p}<.025)$. Newman-Keuls comparisons $(\alpha=.05)$ revealed that the two groups which experienced evaluative feedback during training continued to exhibit faster RTs than the control group during testing but did not differ significantly from each other. Overall RT increased significantly with time on watch during testing $(\mathrm{F}=11.29$, df $=2 / 144, \mathrm{p}<.001)$; there was, however, no significant Groups by Periods interaction $(\mathrm{F}<1)$.

\section{DISCUSSION}

Consistent with previous investigations, KR was found to facilitate performance during training and to have residual properties which, in this case, persisted throughout a 45 -min testing session. However, the performance standards upon which KR was based contributed little to these effects. Significant differences between the RTs of the best-KR (high standard) and worst-KR (low standard) groups were not observed in any phase of the study.
The duration of the training phase was shorter than the time on task common to most vigilance experiments-usually $1 \mathrm{~h}$ or more. Moreover, while not statistically significant, the performance of the best-KR group tended to improve and that of the worst-KR group to deteriorate as training progressed. Therefore, had the training period been extended, differences between the two KR groups may have become more notable. Nevertheless, Fig. 1 reveals that differences in RT between either $K R$ group and the control at the end of training were twice as great as the difference in RT between the two KR groups themselves. Whatever the effects associated with the KR standards, their contribution to the overall differences in the RTs observed here seem minimal.

Since the performance criteria for the two KR groups differed, the information they received during training regarding the distributions of their RTs was quantitatively different. The information that these groups received also differed qualitatively; Ss in the worst-KR group experienced positive feedback following $91 \%$ of their responses, while those in the best-KR group received negative evaluations $88 \%$ of the time. Clearly, however, these disparities in the nature of KR were not reflected in the performances of the Ss. Such results support an incentive rather than an informational interpretation of the effects of KR; but they do not substantiate the prediction from the goal-setting hypothesis that the effects associated with KR would be related to the performance standards used to regulate feedback.

Facilitation of performance by KR implies some degree of self-evaluation on the part of S. Evidence is accumulating that self-evaluations can serve as the basis for self-rewards and criticisms (Kanfer, 1971) and that these operations have reinforcing properties (Montgomery \& Parton, 1970). Recently Warm et al (1972) have reported that S-controlled feedback (self-evaluations of performance efficiency) was as effective as E-controlled dichotomous $\mathrm{KR}$ in facilitating monitoring behavior, even though there was no significant relationship between the accuracy of self-evaluations and performance efficiency. This result, together with the present finding that only the presence of some meaningful evaluation was necessary for sustained improvement, lead to the suggestion that, in a simple vigilance task, the motivational effects of $\mathrm{KR}$ may reside predominantly in its ability to enhance the self-evaluative activity associated with it.

\section{REFERENCES}

Davies, D. R., \& Tune, G. S. Human vigilance performance. London: Staples Press, 1970.

Kanfer, F. H. The maintenance of behavior by self-generated stimuli and reinforcement. In A. Jacobs and L. B. Sachs (Eds.), The psychology of private events: Perspectives on covert response systems. New York: A cademic Press, 1971.

Locke, E. A. Effects of knowledge of results, feedback in relation to standards, and goals on reaction-time performance. American Journal of Psychology, 1968, 81, 566-574.

Locke, E. A., Cartledge, N., \& Koeppel, J. Motivational effects of knowledge of results: A goal-setting phenomenon. Psychological Bulletin, 1968, 70, 474-485.

Loeb, M., \& Schmidt, E. A. A comparison of different kinds of information in maintaining efficiency on an auditory monitoring task. Ergonomics, 1963, 6, 75-81.

Mackworth, J. F. Vigilance and attention. Baltimore: Penguin Books, 1970.

Montgomery, G. T., \& Parton, D. A. Reinforcing effect of self-reward. Journal of Experimental Psychology, 1970, 84, 273-276.

Warm, J. S., Hagner, G. L., \& Meyer, D. The partial reinforcement effect in a vigilance task. Perceptual \& Motor Skills, 1971, 32, 987-993.

Warm, J. S., Kanfer, F. H., Kuwada, S., \& Clark, J. L. Motivation in vigilance: Effects of self-evaluation and experimenter controlled feedback. Journal of Experimental Psychology, $1972,92,123-127$.

(Received for publication February 6, 1973.) 